\title{
ANÁLISIS DEL COMPORTAMIENTO DEL IMPUESTO A LAS VENTAS CON LA IMPLEMENTACIÓN DEL IMPUESTO NACIONAL AL CONSUMO
}

\author{
(Analysis of behavior of sales tax due to implementation of national consumption tax)
}

\author{
Luisa Fernanda Corredor López
}

Escuela de Contaduría Pública, Universidad Pedagógica y Tecnológica de Colombia, Sogamoso. e-mail: luisa.corredor@uptc.edu.co

(Recibido julio 01 de 2014 y aceptado septiembre 03 de 2014)

\section{Resumen:}

Este artículo presenta una descripción del comportamiento tributario en cuanto a responsables, recaudo y actividades económicas más representativas, tanto en el Impuesto a las Ventas (IVA) para los períodos fiscales 2012 y 2013, como para el Impuesto Nacional al Consumo (INC) durante el período fiscal 2013, con el fin de analizar los efectos que se dieron con la entrada en vigencia de la reforma tributaria del año 2012 sobre estos dos tributos.

Palabras clave: reforma tributaria, impuesto, contribuyentes, recaudo, fiscalización, nuevas obligaciones.

\section{INTRODUCCIÓN}

En Colombia han existido múltiples reformas del sistema tributario con una finalidad específica, pero siempre encaminadas a mejorar los ingresos de la nación y la estructura tributaria y financiera del Estado.

Este artículo expone los efectos que trajo la aplicación de la reforma tributaria del año 2012 con respecto al Impuesto al Valor Agregado (IVA) e Impuesto Nacional al Consumo (INC), las variaciones que se presentaron en su comportamiento tributario en cuanto a responsables y recaudo, durante los períodos fiscales 2012 y 2013 en IVA y 2013 en INC, en la Administración de Impuestos y Aduanas DIAN, Seccional Sogamoso.

\section{Abstract:}

This paper focuses on analyzing the taxation behavior in terms of taxpayers, tax collection and economic activities, both in sales tax for the fiscal periods 2012 and 2013 , and in the national tax to the consumption during the fiscal period 2013. This analysis centers on knowing the effects of the taxation reform in 2012 regarding these two taxes.

Keywords: taxation reform, tax, tax payers, tax collection, supervision, new obligations.

\section{ANÁLISIS DEL COMPORTAMIENTO TRIBU- TARIO DEL IVA E INC EN LAS ACTIVIDADES EXCLUIDAS DE IVA Y GRAVADAS CON INC}

Con la entrada en vigencia de la Ley 1607 de 2012, las actividades de servicio de expendio de comidas y bebidas preparadas en restaurantes, cafeterías, heladerías, fruterías, pastelerías y panaderías para consumo en el lugar, para ser llevadas por el comprador o entregadas a domicilio, y el servicio de expendio de comidas y bebidas alcohólicas para consumo dentro de bares y discotecas, quedaron excluidas del impuesto a las ventas (IVA) y gravadas con el nuevo impuesto nacional al consumo (INC), con una tarifa del $8 \%$. 
Las actividades económicas que directamente se ven afectadas con este cambio de impuesto son seis y se identifican con los siguientes códigos CIIU. Ver Tabla 1.

Tabla 1. Actividades excluidas de IVA y gravadas con

$$
\text { INC }
$$

\begin{tabular}{|c|c|}
\hline $\begin{array}{l}\text { Cód. } \\
\text { CIIU }\end{array}$ & DESCRIPCIÓN \\
\hline 1081 & Elaboración de productos de panadería \\
\hline 5611 & Expendio a la mesa de comidas preparadas \\
\hline 5613 & $\begin{array}{l}\text { Expendio de comidas preparadas en cafete- } \\
\text { rías }\end{array}$ \\
\hline 5619 & $\begin{array}{l}\text { Otros tipos de expendio de comidas prepa- } \\
\text { radas n.c.p. }\end{array}$ \\
\hline 5629 & Actividades de otros servicios de comidas \\
\hline 5630 & $\begin{array}{l}\text { Expendio de bebidas alcohólicas para el } \\
\text { consumo dentro del establecimiento }\end{array}$ \\
\hline
\end{tabular}

* Datos registrados en la Resolución 139 de 2012 de la DIAN

Sobre la información tributaria recolectada para estas seis actividades económicas, se analizó el comportamiento tributario de los contribuyentes en cuanto a número de responsables inscritos y recaudo en el IVA de los años 2012 y 2013 e INC año 2013. De acuerdo con la información recopilada se realizó un comparativo de los datos, a fin de examinar el comportamiento tributario y sus posibles causas, del cual se obtuvieron los siguientes resultados:

\subsection{Efectos en la cantidad de responsables}

En el año 2012, en la DIAN Seccional Sogamoso, había inscritos 2.188 contribuyentes en las actividades objeto de análisis. En el año 2013, después de la reforma tributaria que dejó estas actividades excluidas de IVA y gravadas con INC, había 2.701 contribuyentes inscritos en IVA y 1.180 en INC. El detalle de la cantidad de inscritos por tipo de persona y el porcentaje de incremento se puede observar en la Figura 1.
Como se observa en la Figura 1, en el año 2012 había 2.188 inscritos en IVA, que se supone debieron pasar a ser responsables de INC en el año 2013, pero las cifras muestran que solo el $54 \%$, es decir, 1.180 contribuyentes, actualizó con esta responsabilidad el RUT a diciembre 31 de 2013. Las siguientes son las posibles causas por las cuales el $46 \%$ de inscritos en IVA no migraron a INC:

- Contribuyentes activos que no cumplieron con el deber formal de actualizar el RUT.

- Contribuyentes inactivos que desde hace tiempo no ejercen la actividad económica y no han cumplido con el deber formal de actualizar o cancelar su actividad económica en el RUT.

- Personas naturales que desarrollan actividades bajo franquicia, concesión, regalía, autorización o cualquier otro sistema que implique la explotación de intangibles y que continuaron gravados a la tarifa general de IVA.

- Contribuyentes que tienen actividades combinadas, como: alojamiento en hoteles (5511) y expendio a la mesa de comidas preparadas (5611), y que únicamente tienen la responsabilidad de IVA.

- Contribuyentes que actualizan virtualmente su actividad económica, pero no actualizan o incluyen su responsabilidad en INC.

- Contribuyentes que tienen dos actividades gravadas con INC, pero que no tienen ninguna responsabilidad en el RUT.

- Personas naturales que solicitaron el cese de la responsabilidad en ventas, pero no incluyeron la responsabilidad en INC. 


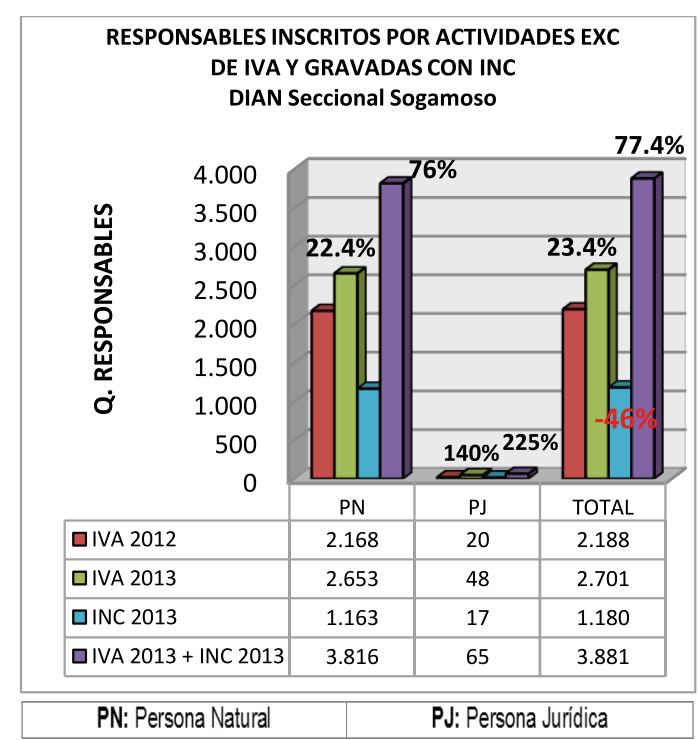

Figura 1. Responsables inscritos por actividades IVA e INC. Datos tomados de DIAN Seccional Sogamoso

Los cambios normativos en las responsabilidades tributarias de las actividades analizadas suponían una disminución en los inscritos en IVA por la migración a INC; sin embargo, el número de inscritos en IVA creció 23.4 $\%$, al pasar de 2.188 en el 2012 a 2.701 en el 2013. Esta situación se presentó por los siguientes factores:

- Mediante la Resolución 000139 expedida por la DIAN el 21 de noviembre de 2012, se reclasificaron los códigos de las actividades económicas. Y, con este cambio, la actividad denominada "Expendio a la mesa de comidas preparadas", que antes se identificaba con el código 5521, quedó con código 5611. Por eso en el análisis se observa el ingreso de nuevos inscritos que en realidad corresponden a contribuyentes antiguos que continúan ejerciendo la misma actividad, pero para la cual cambió el código.

- Personas naturales que desarrollan actividades bajo franquicia, concesión, regalía, autorización o cualquier otro sistema que implique la explotación de intangibles, que continuaron gravados con la tarifa general de IVA y no se cambiaron a INC.

- Nuevas personas jurídicas dedicadas a desarrollar actividades gravadas con INC. Sin embargo, la responsabilidad que tienen a 31 de diciembre de 2013 es la de IVA (11). Se observaron actualizaciones de oficio en el año 2014, cuando se les incluyó la responsabilidad en INC.

- Personas jurídicas cuya actualización de actividad económica fue realizada virtualmente por el representante legal sin asignarle la responsabilidad correspondiente a INC.

- Responsables de INC que desempeñan adicionalmente otra actividad como principal o secundaria gravada con IVA y que están registrados también como responsables en IVA.

- Contribuyentes que tienen una sola actividad económica gravada con INC y tienen las dos responsabilidades en IVA e INC, puesto que no se acercan a tramitar el cese de actividades en ventas.

- Contribuyentes, en su gran mayoría los pertenecientes a régimen simplificado de IVA, que desde hace tiempo no han actualizado el RUT, siguen siendo responsables de IVA.

Las actualizaciones de oficio que hace la DIAN para actualizar las actividades económicas según la Resolución 000139 de 2012, fueron hechas en diferentes tiempos, unas a 31 de diciembre de 2012 y otras durante el transcurso del año 2013, lo que hizo ver un aumento de responsables en IVA 2013.

Las actualizaciones de oficio que hace la DIAN con el fin de incluir nuevas responsabilidades en el RUT, de acuerdo con la actividad económica y la creación de nuevos tributos según lo dispuesto en la reforma tributaria de 2012, únicamente incluye la responsabilidad, no las actualiza ni las retira, quedando el contribuyente con doble responsabilidad, en este caso IVA e INC.

\subsection{Efectos en el recaudo}

En el año 2012, la DIAN Seccional Sogamoso, recaudó por concepto de IVA de las actividades objeto de análisis \$ 3.438 millones. En el año 2013, luego de la reforma tributaria que dejó estas actividades excluidas de IVA y gravadas con INC, recaudó por IVA \$ 1.084 millones y por INC \$ 1.376 millones. (Ver Figura 2). 


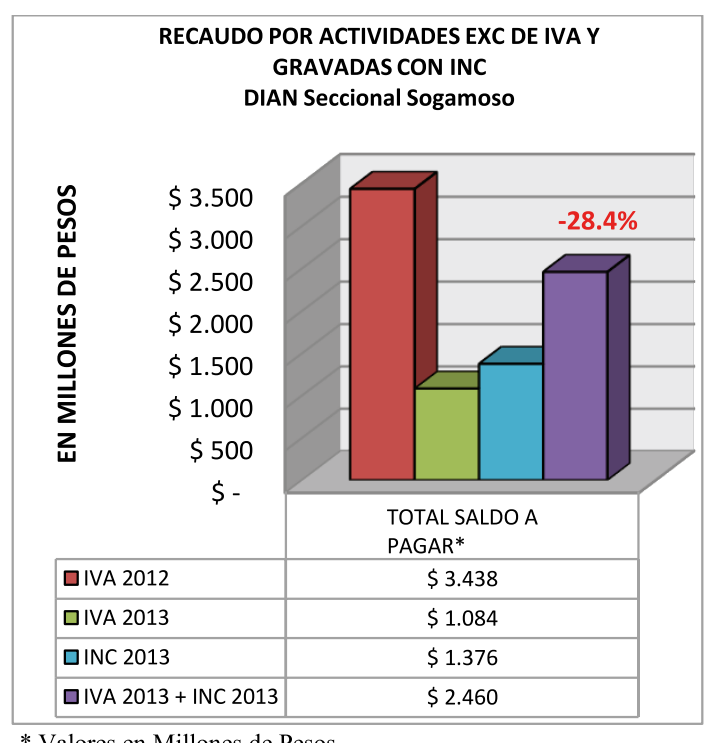

* Valores en Millones de Pesos

Figura 2. Recaudo por actividades IVA e INC. Datos tomados de DIAN Seccional Sogamoso.

Como se observa, en las actividades analizadas, de $\$ 3.438$ millones recaudados en el año 2012 por concepto de IVA, en el año 2013, contados IVA e INC, solo se recaudaron $\$ 2.460$ millones, es decir, en la DIAN Seccional Sogamoso disminuyó el recaudo en $\$ 978$ millones con respecto al año anterior, lo que significa una disminución del $28,4 \%$ en el recaudo.

Entre los hechos identificados como causantes de esta disminución encontramos:

- Disminución de la tarifa de los servicios de expendio a la mesa de comidas preparadas y expendio de bebidas alcohólicas para el consumo dentro del establecimiento, que, de tener una tarifa del $16 \%$ en IVA, pasó al $8 \%$ en INC.

- $\quad$ En el INC 2013 era permitido que las personas jurídicas estuvieran inscritas en régimen simplificado y por tanto no facturaron, ni recaudaron impuesto.

- Los cambios normativos abrieron paso para que personas inscritas en régimen común de IVA se pasaran a régimen simplificado de INC.

Es posible que aprovechando la coyuntura del cambio normativo y los ajustes al RUT, las personas se ubicaron en otras actividades diferentes a las analizadas. Al respecto, también se encuentran contribuyentes que tienen las actividades económicas analizadas pero como actividad secundaria, debido a que no le generan la mayor cantidad de ingresos. Algunos ejemplos se pueden presentar en aquellos contribuyentes que son asalariados o que su actividad principal es la correspondiente a la de su profesión pero que tienen algún establecimiento pequeño donde prestan el servicio ya sea de restaurante o de consumo de bebidas alcohólicas.

Personas que se encuentran omisas en la presentación de declaraciones de INC. Dentro del análisis se observó que de acuerdo con la cantidad de inscritos en INC hubo aproximadamente un $77.2 \%$ de omisos, lo que influyó en el recaudo de este nuevo tributo por causa del desconocimiento y concientización previa del cambio de responsabilidades y nuevas obligaciones en el INC.

Otros factores que tiene una considerable influencia sobre el recaudo son:

- $\quad$ El incremento del mercado informal de comidas y bebidas.

- El no tener descontables, si bien facilita la tributación, la necesidad de sustentar costos es un medio de control del nivel de compras de los contribuyentes que conduce a determinar sus ingresos; es decir, que el no tener descontables da la facilidad a los contribuyentes de no informar la totalidad de sus ingresos.

- Contribuyentes que tienen las dos responsabilidades IVA e INC, pero que solo presentaron una de las dos declaraciones o ninguna de ellas.

- Contribuyentes que aún no tienen conciencia de su responsabilidad tributaria, especialmente aquellos pequeños prestadores de servicio de restaurante, cafeterías, heladerías, fruterías, pastelerías, panaderías, servicio de expendido de bebidas alcohólicas, que no cuentan con asesoría alguna, es decir, no cuentan con asesoría profesional ya que son pequeños comerciantes no obligados a llevar contabilidad.

De acuerdo con el informe de rendición de cuentas de la DIAN del año 2013, el recaudo por IVA interno (decla- 
raciones) disminuyó en un 8,6\%, por lo que se observa que el bajo recaudo no es una situación particular de la Dirección Sogamoso sino de la DIAN a nivel nacional.

Si bien el estudio adelantado arroja que el valor del recaudo en IVA e INC disminuyó en $28.4 \%$, los cambios normativos que obligaron a todos los responsables de las actividades analizadas a facturar sus ingresos, sin importar si pertenecen a régimen simplificado o a régimen común, genera un efecto positivo en renta sobre el cual aún no existen cifras o estudios oficiales.

\section{CONCLUSIONES}

Comparadas y evaluadas las actividades económicas que fueron excluidas de IVA para ser gravadas con INC durante el año 2013, se observó que únicamente 1.180 (54 $\%)$ de los responsables inscritos en IVA 2012 actualizaron su responsabilidad en el RUT; sin embargo se mostró que los inscritos en IVA 2013 con estas mismas actividades aumentó en $23.4 \%$. El recaudo total (IVA $2013+$ INC 2013) por las seis actividades estudiadas disminuyó en $\$ 978$ millones $(28.4 \%)$ teniendo en cuenta el recaudo de IVA 2012, y la actividad con mayor representación en la información fue la identificada con el código CIIU 5611 - Expendio a la mesa de comidas preparadas.

En cuanto a la posibilidad de aumentar el ingreso para el Estado colombiano, en la DIAN Seccional Sogamoso se mostró que sumados los saldos y aportes realizados por los responsables tanto de IVA como de INC durante el período fiscal 2013, respecto a los presentados por IVA en 2012, los indicadores dejan ver que se disminuyó el recaudo producto de la reforma tributaria de 2012.

Por último, la DIAN carece de un sistema de información gerencial que facilite la consulta y análisis de los cambios en la información del RUT. La percepción de la información del RUT es que no es confiable para hacer estudios porque se encuentra desactualizada por parte de los contribuyentes, y las actualizaciones de oficio, al no considerar sino la parte documental y legal, no atienden la realidad económica del contribuyente.

\section{RECOMENDACIONES}

El RUT, como mecanismo único de identificación, ubicación y clasificación de los contribuyentes, es insumo fundamental para la función fiscalizadora y de medición de la DIAN, razón por la cual es indispensable iniciar un proceso de depuración de la información allí registrada, con el fin de acercarla a la realidad fiscal y económica de los contribuyentes de la región.

Promover la integración entre la DIAN y la universidad, para trabajar en pro de dar a conocer y socializar su estrategia de cultura de la contribución, de tal forma que pueda llegar a todos los ciudadanos colombianos, permitiéndole a cada uno el cumplimiento oportuno y voluntario de sus deberes formales, todo ello dentro de un marco constitucional.

Trabajar en conjunto con universidades, colegios, administraciones municipales, cámaras de comercio, juntas de acción comunal o a través de redes sociales realizando campañas, charlas o foros educativos y de sensibilización a la comunidad en general, no solo a los asesores o profesionales de la contaduría pública, acerca de los cambios que se realicen al sistema tributario, con el fin de minimizar riesgos para la administración y los contribuyentes.

Mejorar el sistema de información para el diligenciamiento del formulario del registro único tributario RUT, de tal forma que permita clasificar correctamente al contribuyente, disminuyendo la posibilidad de error en la identificación de responsabilidades tributarias.

\section{AGRADECIMIENTOS}

A los docentes del programa de Contaduría Pública, por guiar y otorgar pautas necesarias para formarnos profesionalmente.

$\mathrm{Al}$ director de este proyecto, C.P. Misael Segura, por su tiempo y dedicación para dirigir, apoyar y trasmitir su conocimiento durante este proceso.

A la DIAN Seccional Sogamoso, por brindarme la oportunidad de desarrollar mi práctica empresarial y permitirme llevar a cabo este proyecto.

Al codirector de este proyecto, C.P. Verónica Sepúlveda Serrano, jefe de División de Gestión de Liquidación de la DIAN Seccional Sogamoso, por aceptar dirigir este proyecto, por su apoyo, tiempo, dedicación, paciencia y por haber compartido su conocimiento abiertamente para el desarrollo de este proyecto. 
Análisis del comportamiento del impuesto a las ventas con la implementación del impuesto nacional al consumo

\section{REFERENCIAS}

Congreso de la República de Colombia (2012, 26 de dic.). Ley 1607 de 2012, por la cual se expiden normas en materia tributaria y se dictan otras disposiciones. Bogotá: Diario Oficial.

Dirección de Impuestos y Aduanas Nacionales -DIAN-. (2012, 21 de nov.). Resolución 139 de 2012, por la cual la Dirección de Impuestos y Aduanas Nacionales DIAN, adopta la Clasificación de Actividades Económicas - CIIU revisión 4 adaptada para Colombia. Bogotá: DIAN.

Dirección de Impuestos y Aduanas Nacionales DIAN. (2013). Rendición de cuentas 2013. Recuperado de http:/www.dian.gov.co/descargas/Rendicioncuentas/2013/RC_2013_DIAN.pdf 